TRANSACTIONS OF THE

AMERICAN MATHEMATICAL SOCIETY

Volume 350, Number 8, August 1998, Pages 3055-3064

S 0002-9947(98)02100-X

\title{
HOMOGENEITY IN POWERS OF SUBSPACES OF THE REAL LINE
}

\author{
L. BRIAN LAWRENCE
}

\begin{abstract}
Working in ZFC, we prove that for every zero-dimensional subspace $S$ of the real line, the Tychonoff power ${ }^{\omega} S$ is homogeneous ( $\omega$ denotes the nonnegative integers). It then follows as a corollary that ${ }^{\omega} S$ is homogeneous whenever $S$ is a separable zero-dimensional metrizable space. The question of homogeneity in powers of this type was first raised by Ben Fitzpatrick, and was subsequently popularized by Gary Gruenhage and Hao-xuan Zhou.
\end{abstract}

\section{INTRODUCTION}

A topological space $X$ is homogeneous if for all $x, y \in X$, there is a homeomorphism $\Phi: X \rightarrow X$ with $\Phi(x)=y$. At the opposite extreme, a space $X$ is rigid if the only homeomorphism of $X$ onto itself is the identity function. Let $\mathbb{R}$ denote the real line with the usual topology. Examples of homogeneous spaces include $\mathbb{R}$ and each of the following subspaces: the open unit interval, the rationals, the irrationals, and the Cantor set. Using transfinite recursion and the axiom of choice, it is also possible to construct rigid subspaces of $\mathbb{R}$ (see the proposition below).

A topological space is zero-dimensional if there is a base for the topology consisting of sets that are simultaneously open and closed. For a subspace $S \subseteq \mathbb{R}, S$ is zero-dimensional iff there is a dense countable subset of $\mathbb{R}$ lying in the complement of $S$; it follows that $S \subseteq \mathbb{R}$ is zero-dimensional iff $S$ is homeomorphic to a subspace of the irrationals (usual topology) (see [3], 348). A rigid subspace of $\mathbb{R}$ is necessarily zero-dimensional.

As usual, we let $\omega$ denote the set of all nonnegative integers, and for a set $S$, we let ${ }^{\omega} S$ denote $\{x: x$ is a function $\& \operatorname{Dom} x=\omega \& \operatorname{Ran} x \subseteq S\}$ (Dom $x$ and $\operatorname{Ran} x$ denote the domain and range of $x$ respectively). We assume that every cartesian product of topological spaces carries the Tychonoff topology of pointwise convergence. A power of a topological space $S$ is a Tychonoff product space where every factor is $S$. Let $\mathbb{P}$ denote the power ${ }^{\omega} \omega$ ( $\omega$ has the discrete topology). Then $\mathbb{P}$ is homeomorphic to the space of irrationals ([3], 348).

Every power of a homogeneous space is of course homogeneous. However, it is possible for homogeneity to hold in a power of a nonhomogeneous factor space. For instance, the Hilbert Cube is homogeneous (O. H. Keller, 1931, [5]); and for any first countable space $S$ in which the isolated points are dense, ${ }^{\omega} S$ is homogeneous (D. B. Motorov, 1989, [9]; this result was obtained independently by Gary

Received by the editors September 7, 1994 and, in revised form, June 1, 1995.

1991 Mathematics Subject Classification. Primary 54B10; Secondary 54E35, 54F99.

Key words and phrases. Real line, separable metric space, zero-dimensional, subspace, product space, power, homogeneous, rigid. 
Gruenhage and Hao-xuan Zhou and was circulated as an unpublished manuscript). As an application of the second result, ${ }^{\omega} S$ is homogeneous if $S$ is a simple convergent sequence (i.e., $S=\left\{\frac{1}{n}: n\right.$ is a positive integer $\} \cup\{0\}$ with the subspace topology from $\mathbb{R}$ ) (homogeneity in this instance also follows from the fact that ${ }^{\omega} S$ is homeomorphic to the Cantor set; see the proof below of the main theorem).

For homogeneity in a power of a subspace $S$ of the real line, the following condition on $S$ is necessary: either every component is a nondegenerate interval, or every component consists of just a single point (a component in a topological space is a maximal connected subset). We are thus led to the following question due to Ben Fitzpatrick (raised in a conversation with Zhou). Is there a subspace $S$ of the Cantor set for which the power ${ }^{\omega} S$ is not homogeneous? The question was popularized (and generalized to first countable zero-dimensional spaces) by Gruenhage and Zhou in the manuscript mentioned above. Gruenhage also suggested it as one of the "New Classical Problems" during a problem session at the 1990 Spring Topology Conference (see [4] for the problem set).

In [2], Fons van Engelen proved that if $S$ is a zero-dimensional metrizable space, then ${ }^{\omega} S$ is homogeneous if either $S$ is first category in itself, or, $S$ contains a dense complete subspace. S. V. Medvedev announced the same result (without proof) in [6]. It follows as a corollary that ${ }^{\omega} S$ is homogeneous if $S$ is a zero-dimensional analytic subspace of $\mathbb{R}$, or, if Martin's Axiom holds and $S$ is a zero-dimensional metrizable space of cardinality less than $c$ (where $c$ denotes the cardinality of the continuum of real numbers).

\section{Results And PROBlems}

(All results are in ZFC.)

Note that a power cannot be rigid since any permutation of the index set induces a homeomorphism on the product space. Suppose that $X$ is a power, and define an equivalence relation on $X$ by: $x \sim y$ iff $\mathcal{C} \ell(\operatorname{Ran} x)=\mathcal{C} \ell(\operatorname{Ran} y)$, where $\mathcal{C} \ell$ is the closure operator in the factor space. Define $X$ to be almost rigid provided that every homeomorphism $\Phi: X \rightarrow X$ setwise fixes each equivalence class.

Proposition. There is a Bernstein subspace $S \subseteq \mathbb{R}$ (i.e., each of $S$ and $\mathbb{R} \backslash S$ intersects every uncountable closed subset of $\mathbb{R}$ ) such that, for each $n \in \omega,{ }^{n} S$ is almost rigid. (Note that the case $n=1$ implies that $S$ itself is rigid.)

Proof. Choose a well ordering in type $c$ for each of: the collection of all uncountable closed subsets of $\mathbb{R}$, and, $\left\{\Phi\right.$ : for some $n \in \omega, \Phi \subseteq{ }^{n} \mathbb{R} \times{ }^{n} \mathbb{R}$ is a homeomorphism where each of $\operatorname{Dom} \Phi$ and $\operatorname{Ran} \Phi$ is a countable dense subspace of ${ }^{n} \mathbb{R}$, and for some $x \in \operatorname{Dom} \Phi, \operatorname{Ran} x \neq \operatorname{Ran} \Phi(x)\}$.

Claim. By transfinite recursion, we can construct sequences of finite subsets of $\mathbb{R}$, $\left\langle s_{\alpha}: \alpha<c\right\rangle$ and $\left\langle t_{\alpha}: \alpha<c\right\rangle$, so that for each $\alpha<c$ :

(1) $\bigcup_{\beta<\alpha} s_{\beta}$ and $\bigcup_{\beta<\alpha} t_{\beta}$ are disjoint;

(2) each of $s_{\alpha}$ and $t_{\alpha}$ intersects the closed set in position $\alpha$ of the well-ordering; and

(3) if $\Phi$ is the homeomorphism in position $\alpha$, and $n \in \omega$ with $\Phi \subseteq{ }^{n} \mathbb{R} \times{ }^{n} \mathbb{R}$, then for $X$ defined to be the $n^{\text {th }}$ power of $\bigcup_{\beta \leq \alpha} s_{\beta}$, either $\Phi$ does not extend to a homeomorphism whose domain and range each includes $X$, or $\Phi$ does extend and for some $x \in X$, either the extension or the inverse of the extension maps $x$ to a point whose range intersects $\bigcup_{\beta \leq \alpha} t_{\beta}$. 
Fix $\alpha<c$, and assume that stage $\beta$ in the recursion has been completed for each $\beta<\alpha$. Choose $\Phi$ and $n$ according to the third clause above. Then we can choose basic open sets $U=\prod_{m<n} U_{m}$ and $V=\prod_{m<n} V_{m}$ in ${ }^{n} \mathbb{R}$ such that:

(1) each $U_{m}$ and $V_{m}$ is an open interval;

(2) $\exists m<n\left[\forall i<n\left(\mathcal{C} \ell\left(U_{m}\right) \cap \mathcal{C} \ell\left(V_{i}\right)=\varnothing\right)\right.$ or $\left.\forall i<n\left(\mathcal{C} \ell\left(V_{m}\right) \cap \mathcal{C} \ell\left(U_{i}\right)=\varnothing\right)\right]$, where $\mathcal{C} \ell$ denotes the closure operator in $\mathbb{R}$; and,

(3) $\Phi[U \cap \operatorname{Dom} \Phi] \subseteq V$.

Let $x \in U$ such that $x$ is $1-1$ and $\operatorname{Ran} x$ is disjoint from the union: $\bigcup_{\beta<\alpha} s_{\beta} \cup$ $\bigcup_{\beta<\alpha} t_{\beta}$. If $\Phi$ extends continuously to $x$, then let $y$ be the value of the extension at $x$, and let $r \in \operatorname{Ran} x \backslash \operatorname{Ran} y$ (this choice is possible since $x$ is $1-1$ and $\operatorname{Ran} x \neq \operatorname{Ran} y$ ). Now choose distinct points $p, q \in \mathbb{R} \backslash\left(\operatorname{Ran} x \cup \operatorname{Ran} y \cup \bigcup_{\beta<\alpha} s_{\beta} \cup \bigcup_{\beta<\alpha} t_{\beta}\right)$ where each belongs to the closed set in position $\alpha$ (this choice is possible by the CantorBendixson Theorem ([3], 85)).

If $\Phi$ does not extend continuously to $x$, or $\Phi$ extends and Ran $y$ intersects $\bigcup_{\beta<\alpha} t_{\beta}$, then let $s_{\alpha}=\{p\} \cup \operatorname{Ran} x$, and let $t_{\alpha}=\{q\}$; otherwise, let $s_{\alpha}=\{p\} \cup \operatorname{Ran} y$, and let $t_{\alpha}=\{q, r\}$.

This completes the recursion. Let $S=\bigcup_{\alpha<c} s_{\alpha}$. Since euclidean spaces are hereditarily separable, each ${ }^{n} S$ is almost rigid by construction.

Remark. The strategy used in the proof of the proposition indicates the obstacle that must be overcome in order to achieve a positive result for homogeneity in the $\omega$ power. Suppose that $S$ is an arbitrary subspace of $\mathbb{P}$, and $x, y \in{ }^{\omega} S$. Then our objective is to construct a homeomorphism $\Phi:{ }^{\omega} \mathbb{P} \rightarrow{ }^{\omega} \mathbb{P}$ so that: $(1) \Phi(x)=y$; and, (2) $\Phi\left[{ }^{\omega} s\right]={ }^{\omega} S$. The second clause is of course where the difficulty lies. Let $B$ denote a countable base for ${ }^{\omega} \mathbb{P}$ that forms a tree under reverse set inclusion. We need to construct an order-preserving $G: B \rightarrow B$ so that we can define $\Phi$ by $\Phi\left(\bigcap_{n \in \omega} U_{n}\right)=$ $\bigcap_{n \in \omega} G\left(U_{n}\right)$ for every maximal chain $\left\langle U_{n}: n \in \omega\right\rangle$ in $B$. The problem then is to guarantee that for each $\left\langle U_{n}: n \in \omega\right\rangle, \bigcap_{n \in \omega} U_{n} \in{ }^{\omega} S$ iff $\bigcap_{n \in \omega} G\left(U_{n}\right) \in{ }^{\omega} S$.

Theorem. Suppose that $S$ is a zero-dimensional subspace of the real line. Then ${ }^{\omega} S$ with the Tychonoff topology of pointwise convergence is homogeneous.

Proof. If $S$ is not compact, then the result follows immediately from Lemmas 1 and 2. Suppose that $S$ is compact, in which case, the argument is standard (but nevertheless included for the convenience of the reader) as follows. By the Tychonoff Product Theorem ([3]), 184), ${ }^{\omega} S$ is compact; we also have that ${ }^{\omega} S$ is a zero-dimensional metrizable space without isolated points. It follows that ${ }^{\omega} S$ is homeomorphic to the Cantor set ([3], 455), which, in turn, is homogeneous.

Remark. For a closed set $T \subseteq \mathbb{P},{ }^{\omega} T$ is either homeomorphic to the Cantor set (if $T$ is compact) or to $\mathbb{P}$ itself (if $T$ is not compact).

Corollary. Suppose that $S$ is a separable zero-dimensional metrizable space. Then ${ }^{\omega} S$ is homogeneous.

Proof. Every separable zero-dimensional metrizable space is homeomorphic to a subspace of $\mathbb{P}([3], 447)$; and the theorem is equivalent to the assertion that for every subspace $S$ of $\mathbb{P},{ }^{\omega} S$ is homogeneous.

Remark. In [1], Alan Dow and Elliott Pearl extend our result by showing that ${ }^{\omega} S$ is homogeneous for every first countable zero-dimensional (Hausdorff) topological space $S$; moreover, they prove that the general case essentially reduces to the construction of this paper. 
Problem 1 (Jan van Mill). Is there in ZFC a rigid subspace of the real line whose square is homogeneous? In [7] van Mill constructed a rigid space whose square is homeomorphic to the Hilbert Cube.

Problem 2. Is it possible to construct a rigid subspace of the real line without using transfinite recursion? (Note that a positive result might be relevant to van Mill's problem.)

Outline of the paper. The proof of the main theorem is presented in three selfcontained stages. The first stage consists of Lemmas 1 and 2 along with the proof of Lemma 1. These lemmas contain the basic strategy and give us our theorem as a corollary. The long-winded statement of Lemma 3 constitutes the second stage, and is essentially a proof of Lemma 2 assuming the existence of a certain kind of tree. The third stage is the recursive construction of the tree. The detailed work of each stage is motivated by the requirements of the preceding stage; we hope that as a result, the key features of our construction will seem natural to the reader.

\section{Strategy}

Definitions. For each $p \in \mathbb{P}$ and $n \in \omega$, define $r=r(p, n) \in \mathbb{P}$ by $r(p, n)(m)=$ $r_{m}=p_{m}=p(m)$ for each $m<n$, and $r_{m}=p_{n}$ for each $m \geq n$.

For each $T \subseteq \mathbb{P}$, define $D=D(T) \subseteq \mathbb{P}$ by $D(T)=\{r(p, n): p \in T \& n \in \omega\}$. Note that $D$ is countable and $T$ is contained in the closure of $D$.

Define a subset $T \subseteq \mathbb{P}$ to be suitable provided that:

(1) $T$ is closed in $\mathbb{P}$;

(2) $\left|\left\{p_{0}: p \in T\right\}\right|=\omega$, where the vertical bars denote the cardinality operator; and,

(3) $D(T) \subseteq T$ (in which case, $D(T)$ is a dense countable subset of $T$ ).

Lemma 1. Suppose that $S$ is a noncompact subspace of $\mathbb{P}$, and $x, y \in{ }^{\omega} S$. Then there are a suitable $T \subseteq \mathbb{P}$ and a homeomorphism $\Psi: \mathbb{P} \rightarrow \mathbb{P}$ such that:

(1) $D(T) \subseteq \Psi[S] \subseteq T$; and,

(2) $\Psi[\operatorname{Ran} x \cup \operatorname{Ran} y] \subseteq D(T)$.

Proof. Note that what we need is simply a change of coordinates. Choose a dense countable subset $D^{\prime} \subseteq S$ with $\operatorname{Ran} x \cup \operatorname{Ran} y \subseteq D^{\prime}$. Order $D^{\prime}$ in type $\omega$ (i.e., as a simple sequence).

For each $n \in \omega$, let $L_{n}=\{s \mid s:[0, n] \rightarrow \omega\}$. Since $S$ is not compact, we can choose $m \in \omega$ so that $\left\{s \in L_{m}: \exists p\left[p \in D^{\prime} \& s \subseteq p\right]\right\}$ is infinite. Define a nested increasing sequence of subsets of $D^{\prime},\left\langle R_{n}: n \in \omega\right\rangle$, by $R_{n}=\left\{p \in D^{\prime}: \exists s \in\right.$ $L_{m+n}[s \subseteq p \& q$ does not extend $s$ for each $q$ that precedes $p$ in the ordering of $\left.\left.D^{\prime}\right]\right\}$. Note that $D^{\prime}=\bigcup_{n \in \omega} R_{n}$. With respect to set inclusion, let $G: \bigcup_{n \geq m} L_{n} \rightarrow$ $\bigcup_{n \in \omega} L_{n}$ be an (onto) order-isomorphism such that for each $n \in \omega$ :

(1) $G\left[L_{m+n}\right]=L_{n}$; and,

(2) For each $p \in R_{n}$, if $s=p \mid[0, m+n+1]$ and $t=G(s)$, then $t_{n+1}=t_{n}$. Define $\Psi: \mathbb{P} \rightarrow \mathbb{P}$ by $\Psi(p)=\bigcup_{n \in \omega} G(p \mid[0, m+n])$. Then $\Psi$ is a homeomorphism. Let $T$ be the closure of $\Psi[S]$ in $\mathbb{P}$. Then $T$ is suitable with $D(T)=\Psi\left[D^{\prime}\right]$.

Lemma 2. Suppose that $T \subseteq \mathbb{P}$ is suitable, and let $D=D(T)$. Suppose further that $x, y \in{ }^{\omega} D$. Then there is a homeomorphism $\Phi:{ }^{\omega} T \rightarrow{ }^{\omega} T$ such that:

(1) $\Phi(x)=y$; and, 
(2) for every $z \in{ }^{\omega} T$, Ran $z \cup D=\operatorname{Ran} \Phi(z) \cup D$; it follows that for every subspace $S \subseteq T$ with $D \subseteq S, \Phi\left[{ }^{\omega} S\right]={ }^{\omega} S$ (use the fact that for every $z \in{ }^{\omega} T, z \in{ }^{\omega} S$ iff $\operatorname{Ran} z \subseteq S$ ).

(Lemma 2 is a corollary of Lemma 3.)

\section{Reduction of Lemma 2 to Lemma 3}

Definitions. Let $N:{ }^{\omega \times \omega} \omega \rightarrow{ }^{\omega} \mathbb{P}$ be the natural map defined by $N(u)=\left\langle z_{k}: k \in\right.$ $\omega\rangle$ where $z_{k}=\langle u(k, i): i \in \omega\rangle$.

For each $k \in \omega$, let $\Lambda_{k}=\left\{\sigma \mid \sigma:[0, k] \times[0, k] \rightarrow \omega \& \exists z \in{ }^{\omega} T\left[\sigma \subseteq N^{-1}(z)\right]\right\}$, where $T$ is the set specified in the hypothesis of Lemma 2. Let $\Sigma=\bigcup_{k \in \omega} \Lambda_{k}$.

For each $k \in \omega$, let $\lambda_{k} \in \Lambda_{k}$ with $\lambda_{k} \subseteq N^{-1}(x)$ if $k$ is even, and $\lambda_{k} \subseteq N^{-1}(y)$ if $k$ is odd, where $x$ and $y$ are the points specified in the hypothesis of Lemma 2 .

Let $\mathcal{F}=\{f: f$ is a function $\& f \subseteq \omega \times \omega \& f$ is $1-1\}$.

Lemma 3. There is a partial ordering $\preceq$ of $\Sigma$ (as usual, we mean that $\preceq$ is a relation on $\Sigma$ that is reflexive, anti-symmetric, and transitive), and an order-preserving function

$$
h: \Sigma \rightarrow\{f \in \mathcal{F}: f \text { is finite }\}(\text { i.e., } \sigma \preceq \tau \text { implies } h(\sigma) \subseteq h(\tau))
$$

satisfying each of the five conditions listed below.

Condition 1. The ordered pair $\langle\Sigma, \preceq\rangle$ is a tree (i.e., for each $\tau \in \Sigma,\{\sigma \in \Sigma: \sigma \prec$ $\tau\}$ is well-ordered by $\prec$ ) where the height is $\omega$ (i.e., every point has only a finite number of $\prec$ predecessors, but there does not exist a uniform upper bound), and for each $k \in \omega$ :

(1) $\Lambda_{k}$ is the $k^{t h}$ level (i.e., the set of all points with exactly $k$-many $\prec$ predecessors);

(2) $\lambda_{k} \prec \lambda_{k+1}$; and,

(3) if $\sigma \in \Lambda_{k}$ and $\mu \in \Lambda_{k+2}$, then $\sigma \preceq \mu$ iff $\sigma \subseteq \mu$.

Definitions. Define a branch of $\langle\Sigma, \preceq\rangle$ to be a function $\eta$ : $\omega \rightarrow \Sigma$ such that for each $k \in \omega, \eta_{k} \in \Lambda_{k}$ and $\eta_{k} \prec \eta_{k+1}$.

Define the map $\Phi:{ }^{\omega} T \rightarrow{ }^{\omega} T$ by $\Phi(z)=z^{\prime}$, where $z=N\left(\bigcup_{k \in \omega} \eta_{2 k}\right)$ and $z^{\prime}=$ $N\left(\bigcup_{k \in \omega} \eta_{2 k+1}\right)$, for each $\prec$ branch $\eta: \omega \rightarrow \Sigma$.

Corollary to Condition 1. The map $\Phi$ is a homeomorphism with $\Phi(x)=y$.

Remark. Define a metric $d$ on ${ }^{\omega} \mathbb{P}$ by $d\left(z, z^{\prime}\right)=\frac{1}{k+1}$ where $k \in \omega$ is the least index for which $N^{-1}(z)\left|[0, k] \times[0, k] \neq N^{-1}\left(z^{\prime}\right)\right|[0, k] \times[0, k]$. Then with respect to $d$, each of $\Phi$ and $\Phi^{-1}$ is uniformly continuous.

Definition. For each $\prec$ branch $\eta: \omega \rightarrow \Sigma$, define $F(\eta) \in \mathcal{F}$ by $F(\eta)=\bigcup_{k \in \omega} h\left(\eta_{k}\right)$.

Condition 2. Suppose $\eta: \omega \rightarrow \Sigma$ is a $\prec$ branch. Let $z=N\left(\bigcup_{k \in \omega} \eta_{2 k}\right)$, and let $z^{\prime}=\Phi(z)$. Then for every $n \in \operatorname{Dom} F(\eta), z_{n}=z_{m}^{\prime}$ where $m=F(\eta)(n)$; it follows that if $\operatorname{Dom} F(\eta)=\omega$ (resp., $\operatorname{Ran} F(\eta)=\omega$ ), then $\operatorname{Ran} z \subseteq \operatorname{Ran} \Phi(z)$ (resp., $\operatorname{Ran} \Phi(z) \subseteq \operatorname{Ran} z$ ); so in particular, if $F(\eta)$ is a permutation, then $\operatorname{Ran} z=$ $\operatorname{Ran} \Phi(z)$.

Definition. For each finite $f \in \mathcal{F}$ and each $k \in \omega \backslash\{0\}$ with $f \subseteq[0, k-1] \times[0, k-1]$, define the extension $E(f, k) \in \mathcal{F}$ by $E(f, k)=f \cup\{\langle i, j\rangle\}$ where:

(1) $j=k$ and $i$ is the least element in $\omega \backslash \operatorname{Dom} f$ if $k$ is even; and, 
(2) $i=k$ and $j$ is the least element in $\omega \backslash \operatorname{Ran} f$ if $k$ is odd. Note that if $f$ is the empty function, then $E(f, 0)=\{\langle 0,0\rangle\}$.

Condition 3. If $\sigma$ is a root of $\langle\Sigma, \preceq\rangle$ or if $\sigma \in \operatorname{Ran} \lambda$, then $h(\sigma)$ is the empty function. If $\tau \in \Lambda_{k}$ with $k>0$, and $\sigma$ is the immediate predecessor of $\tau$, then either $h(\tau)=h(\sigma)$ or $h(\tau)=E(h(\sigma), k-1)$.

Definition. Define $\Delta=\left\{\tau \in \Sigma: \tau \in \Lambda_{k}\right.$ with $k>0$, and $h(\tau)=E(h(\sigma), k-1)$ where $\sigma$ is the immediate $\prec$ predecessor of $\tau$ \}.

Condition 4. If $\sigma \in \Sigma \backslash \Delta$, and $\tau$ is an immediate $\prec$ successor of $\sigma$ that is not in $\operatorname{Ran} \lambda$, then $\tau \in \Delta$.

Corollary to Condition 4. Suppose $\eta: \omega \rightarrow \Sigma$ is a branch distinct from $\lambda$. Suppose further that $\left\{\eta_{2 k}: k \in \omega\right\}$ (resp., $\left.\left\{\eta_{2 k+1}: k \in \omega\right\}\right) \backslash \Delta$ is infinite. Then $\operatorname{Dom} F(\eta)$ (resp., $\operatorname{Ran} F(\eta))=\omega$. The dual statement also holds where in the hypothesis we replace infinite with finite, and in the conclusion we interchange domain and range. (The dual statement follows immediately from the definitions of $\Delta$ and $E$.)

In combination, the four results of the first paragraph imply that one of the following must hold:

(1) $F(\eta)$ is a permutation; or,

(2) $\operatorname{Ran} F(\eta)=\omega$ and $\left\{\eta_{2 k}: k \in \omega\right\} \backslash \Delta$ is finite; or,

(3) $\operatorname{Dom} F(\eta)=\omega$ and $\left\{\eta_{2 k+1}: k \in \omega\right\} \backslash \Delta$ is finite.

Condition 5. Suppose $\eta: \omega \rightarrow \Sigma$ is a $\prec$ branch. Let $z=N\left(\bigcup_{k \in \omega} \eta_{2 k}\right)$, and let $z^{\prime}=\Phi(z)$. Suppose further that $\left\{\eta_{2 k}: k \in \omega\right\}$ (resp., $\left.\left\{\eta_{2 k+1}: k \in \omega\right\}\right) \backslash \Delta$ is finite. Then for every $n \notin \operatorname{Dom} F(\eta)$ (resp., $\operatorname{Ran} F(\eta)$ ), $z_{n}$ (resp., $z_{n}^{\prime}$ ) is eventually constant.

\section{Auxiliary CONCEPts}

The following concepts of correspondence according to $f$ and an $f$ extension, for $f \in \operatorname{Ran} h$, are at the heart of our construction of the tree. In our application of these concepts, we will take the function $f$ to be $h(\tau)$ (see below). With this assignment in mind, the definitions are then motivated by the objective of ultimately satisfying the five conditions of Lemma 3.

Hypothesis. Throughout this section, suppose that $k \in \omega \backslash\{0\}$ and $f \in \mathcal{F}$ with $f \subseteq[0, k-1] \times[0, k-1]$.

Definitions. Suppose $\tau \in \Lambda_{j}$ for some $j \geq k-1$, and $\mu \in \Lambda_{j+1}$. Then we will say that $\tau$ and $\mu$ correspond according to $f$ provided that: if $j$ is even (resp., odd), then $\forall n \in \operatorname{Dom} f \forall m \leq j[\tau(n, m)=\mu(f(n), m)]$ (resp., $[\tau(f(n), m)=\mu(n, m)]$ ).

Define $A(f, k)=\left\{\langle\sigma, \tau, \mu, \nu\rangle \in \Lambda_{k-1} \times \Lambda_{k} \times \Lambda_{k+1} \times \Lambda_{k+2}\right.$ :

(1) $\sigma \subseteq \mu \& \tau \subseteq \nu$;

(2) if $k$ is even (resp., odd), then $\forall n<k$ and $n \notin \operatorname{Ran} f$ (resp., Dom $f$ )

$$
[\sigma(n, k-1)=\mu(n, k)=\mu(n, k+1)] ;
$$

and,

(3) each of the pairs $\tau$ and $\mu$ and $\mu$ and $\nu$ correspond according to $E(f, k)$ (in fact, since $\tau \subseteq \nu$, the second correspondence implies the first)\}. 
Lemma 4. Suppose $\langle\sigma, \tau, \nu\rangle \in \Lambda_{k-1} \times \Lambda_{k} \times \Lambda_{k+2}$ such that $\sigma$ and $\tau$ correspond according to $f$ and $\tau \subseteq \nu$ (see the hypothesis for this section). Then there is a unique function $\xi:[0, k] \times[0, k+1] \rightarrow \omega$ such that for every $\mu \in \Lambda_{k+1},\langle\sigma, \tau, \mu, \nu\rangle \in A(f, k)$ iff $\mu \supseteq \xi$.

Proof. To prove existence we must show that the three clauses in the definition of $A$ do not impose incompatible constraints on $\mu$. The inclusion $\sigma \subseteq \mu$ (clause (1)) and the correspondence requirement (clause (3)) are consistent as a result of the correspondence between $\sigma$ and $\tau$ according to $f$ and the inclusion $\tau \subseteq \nu$. For the second clause, note that if $k$ is even (resp., odd) and $n<k$, then $n \notin \operatorname{Ran} f$ (resp., $\operatorname{Dom} f$ ) iff $n \notin \operatorname{Ran} E(f, k)$ (resp., Dom $E(f, k)$ ).

Uniqueness of the value $\xi(n, m)$, in the case $k$ is even (resp., odd), follows from the correspondence between $\mu$ and $\nu$ according to $E(f, k)$ if $n \in \operatorname{Ran} E(f, k)$ (resp., Dom $E(f, k)$ ), and otherwise, from $\sigma \subseteq \mu$ and the second clause in the definition of A.

Definition and terminology. For each $\langle\sigma, \tau, \mu, \nu\rangle \in A(f, k)$, we will call $\{\sigma, \tau, \mu, \nu\}$ an $f$ extension of $\{\sigma, \tau, \nu\}$; and, if $\langle\tau, \nu\rangle \in \Lambda_{0} \times \Lambda_{2}$ with $\tau \subseteq \nu$, then we define $\{\tau, \mu, \nu\}$ to be a ground extension of $\{\tau, \nu\}$ provided that $\mu \in \Lambda_{1}, \mu(0,0)=$ $\nu(0,0)=\tau(0,0)$, and $\mu(0,1)=\nu(0,1)$.

Note that we are extending sets as opposed to orderings. In our construction of the tree, for each chain of the form $\sigma \prec \tau \prec \nu$, we choose $\mu$ so that $\langle\sigma, \tau, \mu, \nu\rangle \in$ $A(h(\tau), k)$ and either $\sigma \prec \tau \prec \mu$ (but not necessarily $\mu \prec \nu$ ) (Recursive Case 1 below) or $\sigma \prec \tau \prec \mu \prec \nu$ (Recursive Case 2).

\section{Construction of the tree}

Preliminary definitions. Let $\rho: \omega \rightarrow \Sigma$ such that:

(1) $\left|\rho^{-1}(\sigma)\right|=\omega$ for each $\sigma \in \Sigma$;

(2) if $\sigma \subseteq \tau$ and $m$ (resp., $n$ ) is the least point in $\rho^{-1}(\sigma)$ (resp., $\rho^{-1}(\tau)$ ), then $m \leq n$; and,

(3) for each $k \in \omega$ and each $\sigma \in \Lambda_{k}$, if $m, n \in \omega$ with $m<n$ and $\rho(m)=\rho_{m}=$ $\rho_{n}=\sigma$, then there exists $\tau \in \Lambda_{k+2}$ and $i \in \omega$ with $\sigma \subseteq \tau, m<i<n$, and $\rho_{i}=\tau$.

For each $n \in \omega$, define $M_{n} \subseteq \omega$ as follows. Let $k, m \in \omega$ such that $\rho_{n} \in \Lambda_{k}$ and $m$ is the least integer greater than $n$ with $\rho_{m}=\rho_{n}$. Then $M_{n}=\{i \in \omega: n<i<$ $\left.m \& \rho_{i} \in \Lambda_{k+2} \& \rho_{i} \supseteq \rho_{n}\right\}$.

Note that by the choice of $\rho$, each $M_{n}$ is nonempty, and $\left\{M_{n}: n \in \omega\right\}$ is a partition of $\left\{i \in \omega: \rho_{i} \in \Sigma \backslash\left(\Lambda_{0} \cup \Lambda_{1}\right)\right\}$.

Definition of the tree. We will now construct the tree, beginning with the definition in three explicit cases:

(1) if $\tau \in \Lambda_{0}$, then $\tau$ is a $\prec$ root and $h(\tau)$ is the empty function;

(2) If $\tau \in \Lambda_{1} \backslash\left\{\lambda_{1}\right\}$, then the immediate $\prec$ predecessor of $\tau$ is the unique $\sigma \in \Lambda_{0}$ such that $\sigma \subseteq \tau$, and $h(\tau)=\{\langle 0,0\rangle\}=E(h(\sigma), 0)$; and,

(3) for each $k \in \omega, \lambda_{k}$ is the immediate $\prec$ predecessor of $\lambda_{k+1}$, and $h\left(\lambda_{k}\right)$ is the empty function.

We will define the following simultaneously by recursion on $n \in \omega$ :

(1) $\theta_{n}: M_{n} \rightarrow \Lambda_{k+1}$ where $\rho_{n} \in \Lambda_{k}$;

(2) $h(\tau) \in \mathcal{F}$ with $h(\tau) \subseteq[0, k-1] \times[0, k-1]$, where $\tau \in \Lambda_{k}$, for $\tau=\rho_{n}$ and each $\tau \in \operatorname{Ran} \theta_{n}$ (if $h(\tau)$ has not already been defined explicitly); and, 
(3) the immediate $\prec$ predecessor of $\rho_{n}$ (again, assuming the predecessor does not have an explicit definition).

Fix $m \in \omega$ and assume that stage $n$ in the recursion has been completed, according to the three clauses of the preceding paragraph (to be referred to as the first part of the recursion hypothesis), for each $n<m$.

Also, as a second part of the recursion hypothesis, suppose that for each $n<m$ :

(1) (Correspondence) if $\tau=\rho_{n}$ is not a root and $\sigma$ is the immediate $\prec$ predecessor of $\tau$, then:

(i) $h(\sigma)$ is defined and $h(\sigma) \subseteq h(\tau)$; and,

(ii) $\sigma$ and $\tau$ correspond according to $h(\tau)$;

(2) (Extension) for each $i \in M_{n}:\{\sigma, \tau, \mu, \nu\}$ is an $h(\tau)$ extension of $\{\sigma, \tau, \nu\}$ (i.e., $\langle\sigma, \tau, \mu, \nu\rangle \in A(h(\tau), k))$ where $\tau=\rho_{n} \in \Lambda_{k}, \sigma$ is the immediate $\prec$ predecessor of $\tau$ (assuming $\tau$ is not a root), $\nu=\rho_{i}$, and $\mu=\theta_{n}(i)$; and if $\tau$ is a root, then $\{\tau, \mu, \nu\}$ is a ground extension of $\{\tau, \nu\}$; and

(3) (Disjointness) $\operatorname{Ran} \theta_{n}$ is disjoint from the union: $\bigcup_{i<n} \operatorname{Ran} \theta_{i} \cup \operatorname{Ran} \lambda \cup$ $\left\{\rho_{i}: i \leq n\right\}$.

Part One of Stage $m$. First we will define the immediate $\prec$ predecessor of $\rho_{m}$, and $h\left(\rho_{m}\right)$, using two cases. Let $\tau=\rho_{m}$. For this part of stage $m$, we can assume that $\tau \in \Lambda_{k}$ for some $k \geq 2, \tau \notin \operatorname{Ran} \lambda$, and $\tau \neq \rho_{n}$ for each $n<m$.

Recursive Case 1. For some $n<m$ and $i \in M_{n}, \tau=\theta_{n}(i)$; by the Disjointness Clause of the recursion hypothesis, there can be at most one $n$ with this property. Let $\sigma=\rho_{n}$. Then define $\sigma$ to be the immediate $\prec$ predecessor of $\tau$, and define $h(\tau)=E(h(\sigma), k-1)$ (defining $h(\tau)$ at this point is in fact redundant; by our recursion hypothesis, $h(\tau)$ has already been defined since $\tau$ is a point in $\operatorname{Ran} \theta_{n}$ ).

Recursive Case 2. For each $n<m, \tau \notin \operatorname{Ran} \theta_{n}$. By the choice of $\rho$, we can choose $n<m$ with $m \in M_{n}$; moreover, this choice is unique. Let $\sigma=\theta_{n}(m)$. Then define $\sigma$ to be the immediate $\prec$ predecessor of $\tau$, and let $h(\tau)=h(\sigma)$.

Part Two of Stage $m$. For the second part of stage $m$, we return to an arbitrary $\rho_{m}$. Let $k \in \omega$ with $\rho_{m} \in \Lambda_{k}$. Using preservation of the Correspondence Clause, we claim that it is now possible to choose $\theta_{m}: M_{m} \rightarrow \Lambda_{k+1}$ according to the Extension and Disjointness Clauses. By Lemma 4 (or the definition of ground extension if $\rho_{m}$ is a root): for each $\mu \in \Lambda_{k+1}$, satisfaction of the Extension Clause in the choice of $\theta_{m}$ constrains the values of $\mu$ only at arguments belonging to $[0, k] \times[0, k+1]$; so for each $i \in M_{m}$, there are infinitely many choices for $\mu=\theta_{m}(i)$ satisfying the Extension Clause, whereas only finitely many of these are excluded by the Disjointness Clause. For each $\mu \in \operatorname{Ran} \theta_{m}$, define $h(\mu)=E(h(\tau), k)$ where $\tau=\rho_{m}$. Note that this is consistent with the definition of $h$ in Recursive Case 1 above.

This completes the definition of $\theta, h$, and the immediate $\prec$ predecessor function; the reader can verify the preservation of each clause of each part of the recursion hypothesis.

\section{Proof of Lemma 3}

The five conditions can be verified using the following observations.

Fact 1. For each $k \in \omega$ and $\tau \in \Lambda_{k+1}$, the immediate $\prec$ predecessor of $\tau$ belongs to $\Lambda_{k}$. For a proof when $\tau \in \Lambda_{1} \cup \operatorname{Ran} \lambda$, use the second and third explicit cases in the definition of the predecessor function; otherwise, use the first clause in the 
first part of the recursion hypothesis. From Fact 1 and the first explicit case in the definition of the predecessor function, it follows that $\langle\Sigma, \preceq\rangle$ is a tree where the height is $\omega$, and for each $k \in \omega, \Lambda_{k}$ is the $k^{t h}$ level.

Fact 2. For each $\sigma \in \Sigma \backslash \Lambda_{0}$, the immediate successor set of $\sigma$ is the union of a finite set and $\bigcup\left\{\operatorname{Ran} \theta_{n}: \rho_{n}=\sigma\right\}$. The elements of the second set are immediate successors of $\sigma$ by virtue of Recursive Case 1 and the Disjointness Clause (which excludes $\rho_{i}$, for each $i \leq n$, from membership in $\operatorname{Ran} \theta_{n}$ ). We'll refer to these points as direct successors of $\sigma$; we also define $\tau \in \Lambda_{1}$ to be a direct successor of $\sigma$ if $\sigma$ is a root, $\sigma \subseteq \tau$, and $\tau \neq \lambda_{1}$. Note that if $\sigma$ is a root, then with the possible exception of $\lambda_{1}$, the immediate successors, the direct successors, and the elements $\tau \in \Lambda_{1}$ with $\sigma \subseteq \tau$, all coincide. The elements of the finite (possibly empty) set referred to above are those immediate successors of $\sigma$ that result from Recursive Case 2 (we'll refer to these points as indirect successors of $\sigma$ ), along with at most one point from $\operatorname{Ran} \lambda$. If $\tau$ is an indirect successor of $\sigma$, then for some $m, n \in \omega: \tau=\rho_{m}$ and $\sigma=\theta_{n}(m)$. Since each value of $\theta$ is a finite function, and $\operatorname{Ran} \theta_{n_{1}}$ is disjoint from $\operatorname{Ran} \theta_{n_{2}}$ for $n_{1} \neq n_{2}, \sigma$ can have at most finitely many indirect successors. Note that every immediate successor is direct, indirect, or a member of $\operatorname{Ran} \lambda$, and that these cases are mutually exclusive by the Disjointness Clause.

Fact 3. For each $k \in \omega$, if $\sigma \in \Lambda_{k}$ and $\mu \in \Lambda_{k+2}$, then $\sigma \preceq \mu$ iff $\sigma \subseteq \mu$. Suppose $\mu \in \operatorname{Ran} \lambda$. If $\sigma \subseteq \mu \in \operatorname{Ran} \lambda$, then $\sigma \in \operatorname{Ran} \lambda$; and if $\sigma, \mu \in \operatorname{Ran} \lambda$, then either $\sigma \subseteq \mu \subseteq N^{-1}(x)$ (for $k$ even) or $\sigma \subseteq \mu \subseteq N^{-1}(y)$ (for $k$ odd). Suppose $\mu \notin \operatorname{Ran} \lambda$. Then by Fact 2, $\mu$ is either a direct or indirect successor (of some $\tau$ ), in which case, the result follows from the first clause in the definition of $A$ and the Extension Clause.

Fact 4. By the Correspondence Clause, for all $\sigma, \tau, \in \Sigma, h(\sigma) \subseteq h(\tau)$ and $\sigma$ and $\tau$ correspond according to $h(\tau)$ whenever $\tau$ is an immediate successor of $\sigma$.

Fact 5. For all $i, n \in \omega$ with $i \leq n, \rho_{i} \notin \operatorname{Ran} \theta_{n}$; therefore, the immediate predecessor of each point in $\operatorname{Ran} \theta_{n}$ is determined by Recursive Case 1 . So by the definition of $h, \Delta=\left(\Lambda_{1} \backslash\left\{\lambda_{1}\right\}\right) \cup\left(\bigcup_{n \in \omega} \operatorname{Ran} \theta_{n}\right)=\{\tau \in \Sigma$ : for some $\sigma \in \Sigma, \tau$ is a direct successor of $\sigma\}$. It follows that for each $\sigma \in \Sigma \backslash \Delta$, every successor of $\sigma$ (with the exception of $\lambda_{k+1}$ if $\sigma=\lambda_{k}$ for some $k \in \omega$ ) is direct (the existence of an indirect successor of $\sigma$ implies that for some $n \in \omega, \sigma \in \operatorname{Ran} \theta_{n}$ ).

Fact 6. For each $k \in \omega \backslash\{0\}$ with $k$ even (resp., odd), and each $\langle\sigma, \tau, \mu\rangle \in \Lambda_{k-1} \times$ $\Lambda_{k} \times \Lambda_{k+1}$ with $\sigma \prec \tau \prec \mu$, if $\mu$ is a direct successor of $\tau$ and $f=h(\tau)$, then $\forall n<k$ with $n \notin \operatorname{Ran} f$ (resp., $\operatorname{Dom} f)[\sigma(n, k-1)=\mu(n, k)=\mu(n, k+1)]$ (this follows from the second clause in the definition of $A$, and the Extension Clause).

\section{REFERENCES}

1. A. Dow and E. Pearl, Homogeneity in powers of first countable, zero-dimensional spaces, Proc. Amer. Math. Soc. (to appear).

2. F. van Engelen, On the homogeneity of infinite products, Topology Proc. 17 (1992), 303-315. MR 95b:54044

3. R. Engelking, General Topology, Polish Scientific Publishers, 1977. MR 58:18316b

4. G. Gruenhage, ed., Topology Proc. 15 (1990), 207-208.

5. O. H. Keller, Die homoiomorphie der Kompakten Konvexen Mengen im Hillbertschen Raum, Math. Ann. 105 (1931), 748-758.

6. S. V. Medvedev, Characterizations of h-homogeneous spaces, Interim Report of the Prague Topological Symposium 2 (1987). 
7. J. van Mill, A rigid space $X$ for which $X \times X$ is homogeneous; an application of infinitedimensional topology, Proc. Amer. Math. Soc. 83 (1981), 597-600. MR 82h:54067

8. J. van Mill and G. M. Reed, Eds., Open Problems in Topology, North-Holland, Amsterdam, 1990. MR 92c:54001

9. D. B. Motorov, Homogeneity and $\pi$-bases, Vestnik Moscow Univ. Ser. 1 (1989) No. 4, 31-34. MR 90m:54049

Department of Mathematics, George Mason University, Fairfax, Virginia 22030-4444

E-mail address: blawrenc@osf1.gmu.edu 